\title{
On-chip cell positioning and sorting using contactless methods: a comparison between different force-fields
}

\author{
Frénéa Marie ${ }^{1}$ and Haddour Naoufel ${ }^{2}$ \\ Laboratoire Ampère (UMR CNRS 5005), ${ }^{1}$ Université de Lyon, ${ }^{2}$ Ecole Centrale de Lyon \\ France
}

\section{Introduction}

Controlling the motion of biological cells is one of the most important challenges of biomedical research. Cell positioning and separation are fundamental functions that have been widely studied in the past decades with the increased interest for stakes of micro total analysis system ( $\mu \mathrm{TAS}$ ) development. Major breakthroughs were achieved in this research area, thanks to the continuous improvement of micro fabrication techniques, now offering the possibility to design tools with feature sizes adapted to cell dimensions and to perform integration of cell handling devices. Moreover, micro fluidics has shown considerable promise as a tool for studying biological molecules and cells.

Our aim is to present recent developments in cell handling strategies, by focusing on dielectrophoretic, magnetic, optic and acoustic techniques, which, despite their specificities, present many similarities. To facilitate comparison, our study is deliberately restricted to contactless manipulation strategies. Contact-free manipulation techniques are intrinsically sterile and can provide fine control of exerted force. In the next section, we will present the forces and concepts used with the view of extracting common scientific bases. Then, we will illustrate their respective potential by presenting applications chosen to emphasize advantages and drawbacks of each technique. The characteristics and performances of the four methods will be summarized in the last part.

\section{Contactless microparticle manipulation: forces and concepts}

\subsection{Dielectrophoresis}

Dielectrophoresis (DEP) is defined as the motion of polarized particles subjected to nonuniform electric fields. Dielectrophoretic effects may arise from spatial non-uniformities of both magnitude and phase of AC electric fields, respectively interacting with the in-phase and out-of-phase components of the induced dipole moment (Wang et al. 1994; Wang et al. 1995).

cDEP: As first described by Pohl, conventional dielectrophoresis (cDEP) is the motion of polarized particle arising from the interaction of the electric field with the net induced 
charges at the particle/medium interface(Pohl 1958). It implies a non-uniform spatial distribution of the field magnitude. The time-averaged DEP force experienced by a cell placed in an AC electric field $\bar{E}(\omega)$ can be written as:

$$
<\mathbf{F}(t)>=2 \pi \varepsilon_{m} r^{3} \operatorname{Re}\left[f_{C M}(\omega)\right] \nabla E_{R M S}^{2}
$$

where $r$ represents the cell radius, $\mathrm{E}_{\mathrm{RMS}}$ and $\omega$ are the electric field RMS intensity and angular frequency, while $\mathrm{f}_{\mathrm{CM}}$ is the Clausius-Mossotti factor, determined by the frequencydependant polarisabilities of the particle and its surrounding medium:

$$
f_{C M}(\omega)=\frac{\varepsilon_{p}^{*}(\omega)-\varepsilon_{m}^{*}(\omega)}{\varepsilon_{p}^{*}(\omega)+2 \varepsilon_{m}^{*}(\omega)}
$$

$\varepsilon_{p}^{*}$ and $\varepsilon_{m}^{*}$ are the complex permittivities of the particle and its immersion medium defined as $\varepsilon^{*}=\varepsilon-j \sigma / \omega, \varepsilon$ and $\sigma$ respectively denoting dielectric constant and conductivity.

Due to the field inhomogeneity, the forces acting on the opposite and equal charges of the induced dipole are unbalanced, which gives rise to a net force. Consequently, particles whose polarizability is greater than that of their surrounding medium $\left(\operatorname{Re}\left(f_{C M}\right)>0\right)$ are directed toward high field regions, (pDEP, Fig.1a), whereas particles whose polarizability is less than that of the medium $\left(\operatorname{Re}\left(\mathrm{f}_{\mathrm{CM}}\right)<0\right)$ move to the region of lowest field intensity $(\mathrm{nDEP}$, Fig.1b).

a)

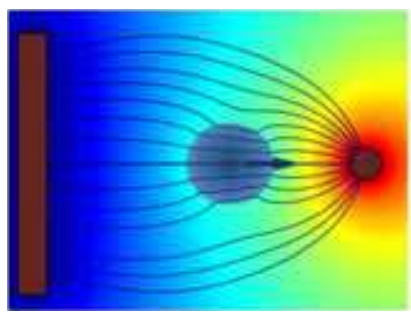

b)

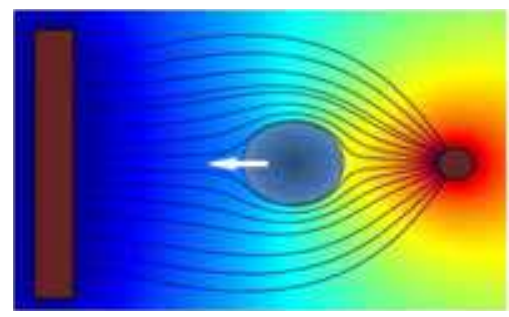

Fig 1. a) positive dielectrophoresis, b) negative dielectrophoresis

twDEP: The twDEP force acts on a particle subjected to a travelling electric field. It is related to the phase non-uniformity of the electric field and arises from the interaction of the travelling field with the phase lagging component of the induced dipole moment. Such a field can be produced by planar electrodes arranged in rows and driven by a polyphase ac voltage. The resulting translational force propels the particle along the electrodes, with or against the field direction, depending on whether $\operatorname{Im}\left(f_{C M}\right)$ is negative or positive, respectively (Jones 2003). In pratice, cDEP and twDEP effects can be observed simultaneously: while the particle translates, it is either pushed above the electrodes (nDEP) or attracted onto them (pDEP), depending on the sign of $\operatorname{Re}\left(f_{C M}\right)$ (Jones 2003).

\subsection{Magnetophoresis}

Magnetophoresis is the magnetic analogue of dielectropheresis and is defined as the translation of para or diamagnetic particles inside a non-uniform magnetic field. A nonspherical particle placed in a homogeneous magnetic field would experience a torque, so as to align with the field, but no translation should be observed. The difference between both 
methods lies in the fact that dielectrophoresis uses electric properties of matter while magnetophoresis exploits its magnetic properties. The magnetic force exerted on a particle depends on the volume of the particle $(V)$, the difference in magnetic susceptibilities $(\Delta \chi)$ between the particle $\left(\chi_{\mathrm{p}}\right)$ and surrounding buffer medium $\left(\chi_{\mathrm{m}}\right)$, as well as the strength and gradient of the applied magnetic field as shown below in equation (3):

$$
F_{\text {mag }}=\frac{2 \pi r^{3}\left(\chi_{p}-\chi_{m}\right)}{3 \mu_{0}} \nabla|B|^{2}
$$

where $r$ is the particle radius, $\mu_{0}$ is the vacuum permeability and $B$ is the magnetic flux density.

Therefore, the net magnetic force exerted on the particle increases with its volume and with the amplitude and the gradient of the field. The net magnetic force increases also with the difference in magnetic susceptibilities $\Delta \chi$ and the sign of the force depends on the sign of $\Delta \chi$. If $\Delta \chi$ is negative, the net magnetic force is negative and the particle is repelled from the maximum region to the minimum region of the magnetic field. Besides, if $\Delta \chi$ is positive, the net force is positive and the particle is attracted to the maximum field region.

\subsection{Optical force}
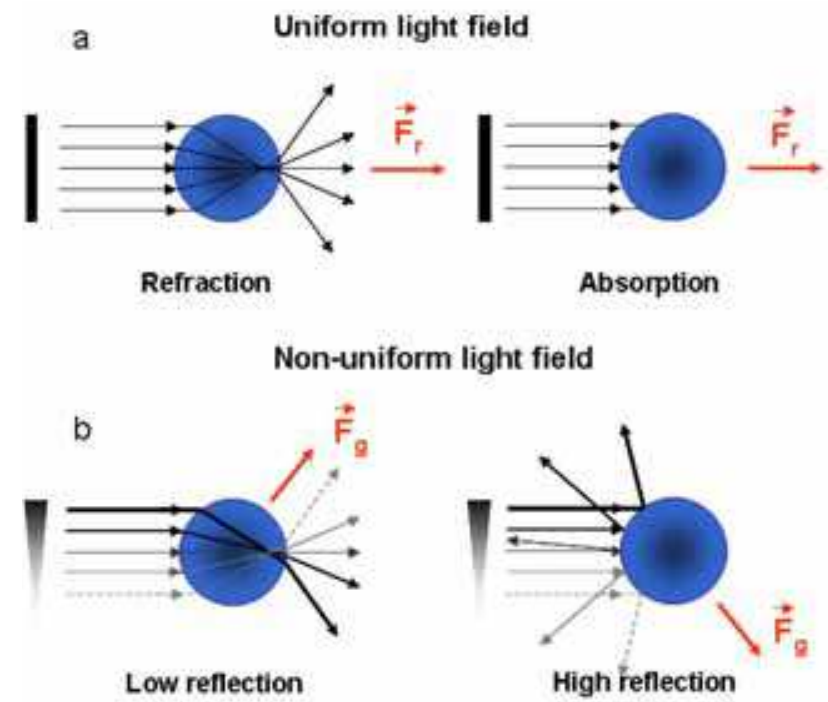

Fig. 2. a. Illustration of scattering force. b. gradient force.

Optical forces can also be used to trap and manipulate particles and biological cells. The light consists of photons having an energy $E=h v$ and a momentum $p=h v / c, h$ denoting the Planck constant $\left(6,626 \times 10^{-34} \mathrm{~J} . \mathrm{s}\right)$ and $v$ the frequency. Thus, when a photon is reflected by an object, it undergoes a variation of momentum that engenders a force on the reflecting 
surface $F=d p / d t=n I / c . n$ is the refractive index of the medium in which the photon propagates, $I$ is the light beam intensity and $c$ is the speed of light. In case where the lightened surface is a dielectric particle, the forces applied on the sphere can be divided into two components. The first is the scattering force $\left(F_{r}\right)$ orientated in direction of light propagation and related to the absorption and the reflection of a uniform light field by the particle (Figure 2.a). The second is the gradient light force $\left(F_{g}\right)$, which is related to interaction of the particle with a non-uniform light field. Photons distributed nonsymmetrically around the centre of the particle result in a gradient force in the direction of the gradient field oblique with respect to the direction of light propagation (Figure 2.b). In the case of a little reflecting particle the gradient force tends to push the object towards the maximum of luminous intensity. However, if the particle is very reflecting, it can be expelled towards the minimal intensity areas.

Under the action of both scattering and gradient forces, a dielectric particle exposed to a focusing laser beam is attracted towards the axe of the beam and propelled in the direction of light propagation. For stable trapping in all three dimensions, the gradient component of the force pulling the particle towards the focal region must exceed the scattering component of the force pushing it away from that region. This condition necessitates a very high field gradient, produced by tightly focusing laser beam using an objective of high numerical aperture NA. Therefore, the force produced by the field gradient can overcome the forces due to gravity, Brownian motion and back-scattered light, resulting in a three-dimensional optical trap. The resulting optical tweezers allow to trap particles and to place them freely on a xy plane. Furthermore, when a highly condensed laser beam is used, the position of the particle can also be controlled in the $\mathrm{z}$ direction. Particles trapped by an optical tweezer can be divided into three regimes determined by the ratio of the incident light's wavelength $\lambda$ to the diameter $\mathrm{D}$ of the irradiated particle. If the particle size is larger than the wavelength of the trapping laser, i.e., the radius $\mathrm{D}>>\lambda$, the particle is in the Mie regime and optical forces can be computed from simple ray optics. If the trapped sphere is much smaller than the wavelength of the trapping laser, i.e., $\mathrm{D}<<\lambda$, the conditions for Raleigh scattering are satisfied and optical forces can be calculated by treating the particle as a point dipole. In this approximation, the scattering and gradient force components are readily separated. When the particle sizes are comparable with the wavelength of the trapping laser, i.e $D \approx \lambda$, neither the ray optic nor the dipole approach is valid. Unfortunately, among objects that lie in this size range $(0.1-10 \mu \mathrm{m})$ are bacteria, yeast, organelles of larger cells and dielectric microspheres used alone or as handles to manipulate other biological objects. The calculation of optical forces requires the solution of Maxwell's equations with the appropriate boundary condition to supply an accurate description. Recently Nieminen et al. developed a computational toolbox implemented in Matlab ${ }^{\circledR}$, for the computational modelling of optical tweezers (Nieminen et al. 2007). The toolbox is designed for the calculation of optical forces and torques, and can be used for both spherical and nonspherical particles, in both Gaussian and other beams. However, in many research works, both the axial and the lateral trapping efficiencies for various configurations of optical tweezers are defined in terms of the constant $Q$ of proportionality in an equation relating the incident laser power $P$ at the focus to the resulting trapping force $F$ (Svoboda et al. 1994). 


$$
F_{\text {Trap }}=\frac{Q n_{m} P}{c}
$$

In equation $4, n_{\mathrm{m}}$ is the refractive index of the suspending medium, $c$ is the speed of light in vacuum and the quantity $n_{\mathrm{m}} . P / c$ is simply the total linear momentum flux of the light beam. $Q$ represents the fraction of power utilized to exert the force. Hence possible values of $Q$ range between 0 and 1 . For plane waves incident on a perfectly absorbing particle, $Q=1$. $Q$ value depends on the NA, laser wavelength, light polarization state, laser mode structure, relative index of refraction, and geometry of the particle.

\subsection{Acoustic force}

When exposed to an ultrasonic field, particles immersed in a liquid experience pressure fluctuations, arising from the displacement of the molecules of the medium. Acoustic forces exerted on the particle originate from acoustic pressure gradients.

The force experienced by particles in an acoustic standing wave is the result of two forces, namely the primary radiation force (PRF), originating from the standing wave, and the Bjerknes force (or secondary radiation force) due to the scattering of the incident sound waves by the particles. In most applications, the main force used is the acoustic PRF, since it is generally orders of magnitude stronger than the secondary forces, rapidly decreasing with interparticle distance. The axial component of the PRF, denoted by $F_{r}$ (equ. 5), acts in the direction of the sound wave propagation and drives the particle toward the pressure nodes or antinodes, depending on the acoustic contrast factor $\phi$ (equ. 6).

$$
F_{r}=-\left(\frac{\pi p_{0}^{2} V_{p} \beta_{m}}{2 \lambda}\right) \phi(\beta, \rho) \sin (2 k x)
$$

where $\phi(\beta, \rho)$ denotes for the acoustic contrast factor defined by :

$$
\phi(\beta, \rho)=\frac{5 \rho_{p}-2 \rho_{m}}{2 \rho_{p}+2 \rho_{m}}-\frac{\beta_{p}}{\beta_{m}}
$$

In the above equations, the parameters $\rho_{p}$ and $\rho_{\mathrm{m}}$ respectively refer to the particle and medium densities, while $\beta_{\mathrm{p}}$ and $\beta_{\mathrm{m}}$ represent their corresponding compressibilities. The axial PRF (equ. 5) is proportional to the acoustic pressure amplitude $\mathrm{p}_{0}$ and to the particle volume $V_{p} \cdot \lambda$ represents the ultrasonic wavelength, $\mathrm{k}$ is the wave number defined by $2 \pi / \lambda$ and $\mathrm{x}$ is the distance from a pressure node. The compressibility $\beta$ is defined as: $\beta=1 /\left(\rho c^{2}\right)$, where $c$ denotes the sound velocity in the corresponding material.

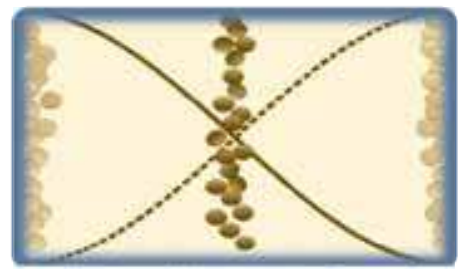

Fig. 3. Generation of an ultrasonic standing wave in a channel which length equals $\lambda / 2$. Particles having a positive acoustic contrast factor $(\phi>0)$ are directed to the chamber midplane toward the pressure node, whereas particles having $\phi<0$ collect at the chamber walls (pressure anti-nodes). 
The technique of acoustic manipulation has been first used in macro-scaled systems, with chamber sizes comprised between a few $\mathrm{mm}$ and a few $\mathrm{cm}$. It had already been applied to separate, filter or agglomerate bio particles from suspensions (Coakley 1997), before gaining increased attention over the last decade, with the recent development of chip integrated strategies (Laurell et al. 2007).

\subsection{Shared principles}

The four types of forces presented above obviously present common characteristics. To start with, they all rely on the existence of a field gradient. As can be deduced from the forces expression, when the field gradient is null (i.e. in uniform fields), the force cancels. In any case, when a particle is placed in a non-uniform field, it experiences a force $F$ that drives it towards the regions where its potential energy $U$ is minimized, according to the following relation: $<\mathrm{F}>=-\nabla<\mathrm{U}>$.

Moreover, particle manipulation can be performed as long as the contrast factor is not null, which indicates that the exploited properties of the particle must differ from the surrounding medium. In the case of DEP, the relevant properties are their frequencydependant dielectric characteristics. When magnetophoresis is employed, the force factor reflects the cell/medium magnetic susceptibility contrast. Acoustophoretic motion is governed by mechanical characteristics of cells and their immersion medium, namely their respective densities and compressibilities. As regards optical cell handling, trapping or pushing effects may be observed, depending on the contrast between cell refractive index, dispersive properties and that of its surroundings. Concerning DEP and optical forces, it may also be pointed out that the DEP contrast factor $\mathrm{f}_{\mathrm{CM}}$ is frequency-dependent and that the refractive indices and dispersions are wavelength-dependent.

\section{Contactless micro particle manipulation: forces and concepts}

\subsection{A determinant parameter: the contrast factor}

The force factor impacts not only the sign of the applied force, but also its magnitude. Accordingly, gaining knowledge about this factor and, when possible, being able to tune it, may be of great interest to control cell motion. To modulate the contrast factor, two strategies can be identified. The first one consists in influencing cell properties, by attaching functionalized beads to their surface, for instance. The second one consists in using the native properties of cells and adapting their immersion buffer composition. Of course, a combination of both these techniques can also be considered.

\section{a. Cell properties}

The dielectric properties of mammalian cells are usually described by the single-shell model, in which the cell interior is represented by an inner sphere of radius $\mathrm{R}$ surrounded by a thin envelope of thickness $\mathrm{d}$, corresponding to the cell membrane. 


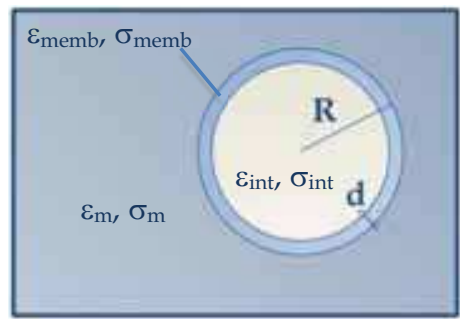

Fig. 4. Single-shell model of a spherical cell. Membrane capacitance, which is defined as $\varepsilon_{\text {memb }} / \mathrm{d}$, ranges from 1.2 to $4 \mu \mathrm{F} / \mathrm{cm}^{2}$ for most mammalian cells, whereas $\sigma_{\text {memb }}$ is usually negligible (Gascoyne et al. 2004). Membrane thickness is generally comprised between 2 and

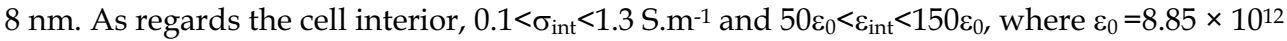
(Gascoyne et al. 1995).

Indeed, the complex permittivity of the cell (which appears in the DEP force factor expression, eqn. 2), reflects several aspects of its structure and composition, as demonstrated by Irimajiri (Irimajiri et al. 1979):

$$
\varepsilon_{p}^{*}=\varepsilon_{m e m b}^{*}\left\lfloor\frac{\left(\frac{R}{R-d}\right)^{3}+2\left(\frac{\varepsilon_{\text {int }}^{*}-\varepsilon_{m e m b}^{*}}{\varepsilon_{\text {int }}^{*}+2 \varepsilon_{m e m b}^{*}}\right)}{\left(\frac{R}{R-d}\right)^{3}-\left(\frac{\varepsilon_{\text {int }}^{*}-\varepsilon_{m e m b}^{*}}{\varepsilon_{\text {int }}^{*}+2 \varepsilon_{m e m b}^{*}}\right)}\right\rfloor
$$

In equation 7 , the subscripts memb and int refer to cell membrane and internal compartment, respectively. As can be understood from this equation, DEP offers a wide range of particle discrimination parameters, including compartment sizes, conductivities and dielectric constants. In the frequency range $5 \mathrm{kHz}-200 \mathrm{MHz}$, cell dielectric properties are mainly related to Maxwell-Wagner polarization resulting from the build up of ions at membrane interfaces under the influence of the electric field (Gascoyne et al. 2004). The time required for the polarization to take place depends on the field frequency, which confers to DEP one of its greatest advantages. Indeed, as the complex permittivity is related to the field frequency, this parameter can be varied to control the direction and magnitude of the DEP force. The evolution of cell dielectrophoretic behavior with respect to frequency may be perceived as the cell fingerprint. Different cell types can therefore be compared on the basis of their DEP spectra. Moreover, alterations in cell membrane integrity or various physiological changes induced in cells following specific treatment may be detected from modified DEP responses (Huang et al. 1999; Ratanachoo et al. 2002; Sudsiri et al. 2007). For example, DEP was used to discriminate between healthy and cancerous cells (Gascoyne et al. 1994) and to detect membrane changes of erythrocytes following malaria infection (Gascoyne et al. 1997; Gascoyne et al. 2002; Gascoyne et al. 2004). Cells exhibiting similar dielectrophoretic phenotypes may also be separated by DEP using marker-specific sorting. $\mathrm{Hu}$ used this technique in combination with nDEP deflection to illustrate affinity-based enrichment of rare bacteria labelled with polymeric beads from an excess of non target bacteria that did not express this marker (Hu et al. 2005). 
The behaviour of a biological cell in presence of a magnetic field depends on its magnetic susceptibility. The magnetic susceptibility $\chi$ is the degree of magnetization of a material in response to an applied field. Magnetic susceptibility is negative for diamagnetic material $(\chi<0)$ and positive for paramagnetic objects $(\chi>0)$. Materials in which $|\chi|<0.01$ are often considered non-magnetic, because their response to an applied magnetic field is negligible. Most cell types are non-magnetic with magnetic susceptibilities very close to that of their usual surrounding medium. Indeed, the susceptibility of vast majority of biological cells is in a narrow range of about $\pm 20 \%$ from that of water, $\chi_{\mathrm{H} 2 \mathrm{O}}=-9.05 \times 10^{-6}$ in SI units (Schenck 2000). Therefore, the difference of magnetic susceptibilities between cells and medium $(\Delta \chi)$ is very small and the magnetic force exerted on the cells is too weak. To our knowledge, there exists a unique type of paramagnetic cells, which are the magnetotactic bacteria, as they enclose magnetite particles. Cell attraction to a magnetic field does not occur solely in paramagnetic cells. For instance, red blood cells have been separated from whole blood by magnetic attraction based on their native susceptibility (Han et al. 2004; Inglis et al. 2006). The deoxygenated red blood cells (erythrocytes), which contain high concentrations of paramagnetic haemoglobin, are diamagnetic cells but have a magnetic susceptibility $\left(\chi_{\mathrm{RBC}}=\right.$ $-6.52 \times 10^{-6}$ in SI units) higher than that of the water and the positive value of $\Delta \chi(\Delta \chi=2.52 \mathrm{x}$ $\left.10^{-6}\right)$ allows attraction of cells to regions of strong magnetic field. Magnetic labelling of nonmagnetic cells can also be performed with commercially available magnetic markers such as superparamagnetic particles, magnetoliposomes or molecular magnetic labels. Detailed overview of magnetic labelling of cells with magnetic markers was documented by Safarik and Pamme. Magnetic labelling of cells allows increasing of their susceptibilities and permits their translation under non-uniform magnetic fields. This strategy has been widely used for separation and purification of cells by a selective magnetic labelling of the cell subpopulation target (Safarik et al. 1999; Pamme 2006).

In the case of optical force, the trapping force depends essentially on the Q-value. As previously mentioned, $\mathrm{Q}$ is determined experimentally and depends on both refraction index and geometry of the cell (Svoboda et al. 1994). These two physical properties respectively linked to the nature of the intracellular content and to the morphometry of the cell both contribute to the optical force. The strength of the gradient force increases with the refractive index of cells. However, the refractive index of most biological cells is in a narrow range of 1.3-1.4 (Balaev et al. 2001; Rappaz et al. 2005; Jin et al. 2006). Refractive indices of different cell types are reported in table 1 . On the contrary, there is a large difference in the size and the shape of cells according to their nature, which can be exploited to distinguish between them (Paterson 2005).

\begin{tabular}{|l|l|}
\hline Cell type & Refractiveindex \\
\hline Escherichia coli bacteria & 1.387 (Balaev et al. 2001) \\
\hline Haemoglobin cytoplasm & $1.387($ Jin et al. 2006) \\
\hline Blood plasma & 1.351 (Jin et al. 2006) \\
\hline Mammalian cells & $1.38-1.41$ (Rappaz et al. 2005) \\
\hline Fibroblasts & $1.358-1.374$ (Rappaz et al. 2005) \\
\hline
\end{tabular}

Table 1. Refractive indices of different cell types

Refractive index of cells can be sensitively changed by labelling them with nano or microparticles having a very different refractive index such as polystyrene and silica 
particles. For instance, Saxena et al. succeeded to enhance optical manipulation of biological cells by labelling them with polystyrene microparticles (Saxena 2003). This technique can be exploited to separate a specific subpopulation of cells. The cells with microspheres attached reacted to the optical landscape more strongly than cells without any attached beads due to the higher refractive index mismatch and resulting optical forces (Dholakia et al. 2007).

Acoustic properties of different cell types are reported in table 2. Most cell types present a higher density and lower compressibility than their usual suspension medium (Tsutsui et al. 2009 ), which gives a positive value of the acoustic contrast factor $\phi$. As a result, the axial PRF drives them towards the standing wave pressure nodes. On the contrary, lipid vesicles and gaz bubbles are generally driven towards the pressure anti-node $(\phi<0)($ Evander et al. 2007; Laurell et al. 2007).

\begin{tabular}{|l|l|l|}
\hline Cell type & Mass density & Compressibility \\
\hline $\begin{array}{l}\text { Escherichia coli } \\
\text { bacteria }\end{array}$ & $1.160 \mathrm{~g} / \mathrm{cm}^{3}($ Godin et al. 2007) & - \\
\hline $\begin{array}{l}\text { Human red blood } \\
\text { cells }\end{array}$ & $\begin{array}{l}1.139 \mathrm{~g} / \mathrm{cm}^{3} \\
(\text { Godin } \text { et al. 2007) }\end{array}$ & $\begin{array}{l}3.38 \times 10^{-10} \mathrm{~Pa}^{-1} \\
(\text { Toubal } \text { et al. } 1999)\end{array}$ \\
\hline HeLa cells & $\begin{array}{l}1.055 \mathrm{~g} / \mathrm{cm}^{3} \\
(\text { Park } \text { et al. } 2008)\end{array}$ & - \\
\hline Water & $0.997 \mathrm{~g} / \mathrm{cm}^{3}$ & $4.48 \times 10^{-10} \mathrm{~Pa}^{-1}$ \\
\hline
\end{tabular}

Table 2. Mechanical properties of different biological particles and of water.

By gaining knowledge about cell properties, one can be able to predict the cell behavior when exposed to the force field under any given operating conditions (frequency, medium properties, etc.). Optimal separation conditions can therefore be estimated, for instance. Common strategies have been developed for the determination of the magnetic and dielectric properties of single cells. They rely on the fact that the force used to actuate cells may also be employed to probe their properties. These techniques imply the measurement of the solution properties (magnetic susceptibility or permittivity and conductivity and, in some cases, viscosity $\eta$ ). The technique of cell and particle tracking velocimetry for cell magnetization measurement was described by Moore (Moore et al. 2000; Moore et al. 2004) and Häfeli (Häfeli et al. 2002). A magnet assembly produces an unidimensional and constant energy density gradient over a region. The magnetic force exerted on a spherical particle therefore becomes:

$$
F_{m}=V \Delta \chi \frac{d}{d x}\left(\frac{B^{2}}{2 \mu_{0}}\right)
$$

The opposing drag force exerted on the migrating particle is $F_{d}=6 \pi r \eta v$. Then, the measured cell speed $v$ may be related to $\Delta \chi$ by the equation:

$$
v=\frac{2 r^{2} \Delta \chi}{9 \eta} \frac{d}{d x}\left(\frac{B^{2}}{2 \mu_{0}}\right)
$$

Magnetophoretic mobility measurements were successfully performed on polystyrene (diamagnetic) beads placed in a solution containing paramagnetic ions. Wanichapichart has used a comparable method to determine cell dielectric properties by measuring the speed of cell translation in between a pair of cylindrical electrodes producing a well-characterized 
electric field (Wanichapichart et al. 2002). When the DEP and drag forces balance, the cell speed $v$ is given by:

$$
v=\frac{\varepsilon_{m} r^{2} \operatorname{Re}\left(f_{C M}\right)}{3 \eta} \nabla E^{2}
$$

For each frequency point, $v$ is plotted against $\nabla E^{2}$ to obtain $\operatorname{Re}\left(\mathrm{f}_{\mathrm{CM}}\right)$. It is therefore possible to deduce the dielectric properties of cell membrane and cytoplasm, by iterative method using the single-shell model presented in the previous section. Despite their similarities, there is a major difference between the techniques employed for magnetic and dielectric characterization of cells presented above, which lies in the fact that the first approach is static, while the second one implies frequency variation.

The technique of electrorotation (ROT) (De Gasperis et al. 1998) has also been intensively used to study the dielectric properties of various cells (Gascoyne et al. 1995; Huang et al. 1995). When a polarisable particle is placed in a rotating AC electric field, a dipole is induced. If the field frequency is sufficiently large, the dipole lags behind the applied field, which gives rise to a torque causing cell rotation. If this phase lag is more than $180^{\circ}$, cell rotates against the field direction. Otherwise the direction of rotation is with the field. ROT is therefore an analogue of twDEP, with electrodes arranged in a circle, rather than in line. At the equilibrium between induced torque and viscous drag, the rotation rate of the particle is:

$$
R(\omega)=-\frac{\varepsilon_{m} \operatorname{Im}\left(f_{C M}\right) E^{2}}{2 \eta}
$$

Curve fitting procedures may be used to obtain the dielectric parameters of the cell, by minimizing the deviation between the experimental ROT spectrum (plot of the rotation rate with respect to the field frequency) and the theoretical spectrum predicted by the singleshell model. Electrorotation data are useful criterions to assess the feasibility of dielectrophoretic cell sorting (Yang et al. 1999).

Regarding optical force, as previously mentioned, $Q$ parameter is the main determinant of trapping force. $Q$ parameter can be experimentally determined using a calibration approach (Saxena 2003). This measure consists in placing the trapped particle in a laminar flow and increasing the hydrodynamic force until the particle escapes from the optical trap at some critical flow velocity. At this critical point, the trapping force (equ. 4) is equal to the drag force $F_{d}=6 \pi r \eta v$. It is therefore possible to calculate $Q$ from measurement of this critical velocity.

\section{b. Medium composition}

The suspension medium composition is of great importance when using any of the contactless manipulation methods described above. Besides the need for operating under physiological conditions, the influence of medium properties on the force factor is also a concern.

Regarding DEP, while the expression of cell permittivity is rather intricate, the external electrolyte permittivity $\varepsilon_{m}^{*}$ is wholly characterized by the bulk dielectric constant and conductivity. Apart from the electric field frequency, one of the variables that can be easily 
adjusted to influence cell dielectric behavior is the medium conductivity. Changing this parameter enables to shift the DEP crossover frequency, which corresponds to the transition frequency between $\mathrm{nDEP}$ and pDEP regimes. Prior to DEP manipulation, cells are often resuspended in low conductivity buffers, in order to prevent Joule heating and undesirable electrothermal effects, and also because only nDEP can be observed in high-conductive media. pDEP-based assays require low-conductivity media, with $\sigma_{m}$ usually ranging from 10 to $200 \mathrm{mS} \mathrm{m}^{-1}$ (Jaeger et al. 2008). In such cases, the medium osmolarity must be adapted by replacing the salts usually found in cell culture media by osmotically equivalent amounts of sugars. Buffers as conductive as culture media $(0.2$ to $2 \mathrm{~S} / \mathrm{m})$ has yet been employed for trapping (Park et al. 2009) or handling (Seger et al. 2004) of biological species using nDEP.

In magnetophoresis, the composition of the surrounding medium is also very important. Indeed, the amplitude and the sign of the magnetic force exerted on the cell depend on the difference of magnetic susceptibilities between cells and medium $(\Delta \chi)$. As we described previously (section 3.2.a) the magnetic susceptibilities of the usual surrounding medium are very close to that of the most biological cell and $\Delta \chi$ is very small. However, it is possible to increase $\Delta \chi$ by increasing magnetic susceptibility of the medium. Indeed, the addition of paramagnetic molecules (such as $\mathrm{Mn} 2+$ or $\mathrm{Gd} 3+$ ions) to the medium increases its susceptibility (Kimura et al. 2004; Winkleman et al. 2004; Kimura et al. 2005). As a result, the obtained $\Delta \chi$ is negative with a large value and the non-magnetic cells experience a negative force that repels them form the magnetic field to the region of minimum field. This technique eliminates the need for cell labelling with magnetic markers for magnetic cell manipulation.

In the case of optical force, since biological cells are usually suspended in aqueous media that have almost the same refractive index, the dependence of optical trapping force on refractive index of the surrounding medium can rarely be exploited to achieve higher trapping forces (Sato et al. 1991).

As previously mentioned, in an aqueous medium, most solid particles have a positive acoustic contrast factor $\phi$. Yet, Gupta et al. demonstrated that two particles with different compressibility or density could be separated by adjusting the medium properties in such a way that the sign of $\phi$ became different for the two particles (Gupta et al. 1995). A similar approach was used by Petersson to enable the fractionation of red cells, platelets, and leukocytes using a differential separation method based on the PRF force magnitude (see section 4.2). Cesium chloride was added into the suspending medium to modify its density and therefore reinforce the contrast between the cell $\phi$ factors (Petersson et al. 2007).

\subsection{Field generation}

High electric field gradients required for efficient cDEP cell handling can be obtained by using metal microelectrodes of appropriate design, fabricated via standard photolithography processing. Interelectrode gap reduction enables strong electric field generation without the need for applying high voltages. Various electrode designs are described in the literature, including large arrays of interdigitated rectangular electrodes (Inoue et al. 1988; Price et al. 1988; Burt et al. 1990; Pethig et al. 1992) and quadrupole electrode microstructures. Both bipolar and quadrupolar electrode configurations were investigated. On the one hand, the choice of bipolar structures simplifies the fabrication of large electrode arrays. On the other hand, quadrupole electrode structures provide 
increased focusing of cells under nDEP (Huang et al. 1991; Frénéa et al. 2003). 3-D field cages enabling accurate single cell trapping were also obtained using octupole arrangement of electrodes (Müller et al. 1999).

An alternative to electrode-based DEP is the technique of insulator-based DEP (iDEP), first employed by Masuda in the design of a cell fusion device (Masuda et al. 1989). It consists in using insulating features to create constrictions in the electric field produced by remote electrodes. It offers the advantage to solve problems inherent in the use of microelectrode, such as electrode deterioration due to electrolysis in the lower frequency range. The accessible frequency range can therefore be widened. This technique also owes its success to the advantages provided by the use of insulating materials, such as plastics, allowing lowcost and rapid fabrication technology (Lapizco-Encinas et al. 2004). A drawback is that a high-voltage power supply is needed to power the distant electrodes.

High magnetic field gradients can be obtained by designing micro magnet and micro electromagnet patterns on silicon or glass substrates. Generally, micro magnets are fabricated with ferromagnetic materials such as samarium cobalt $(\mathrm{SmCo})$ or neodymium iron boron $(\mathrm{NdFeB})$ via standard photolithography processing (Pamme 2006). Integrated micro-electromagnets can be manufactured by depositing micro-coil of conducting material such as cooper, gold or aluminium. Lee et al. have built several arrays of microelectromagnetic coils for cell manipulation. Thanks to controlling independently each microelectromagnetic coil with integrated circuit, they succeeded to turn on and off individually each coil, allowing the displacement of cells to a targeted location (Lee et al. 2004). Ramadan et al. described various designs of micro-coils to obtain high magnetic field gradients (Ramadan et al. 2009). This kind of systems requires utilization of a cooling system to keep the temperature of the surrounding medium at a survivable level for the cells. This makes the device less suitable for experiments that last for hours than other devices that do not generate heat during operation. Another approach to avoid heating problems associated to micro-electromagnetic coils and obtain precise control and strong gradients consists on using micro-patterns with magnetically susceptible features such as nickel, permalloy or soft iron. Then, micro-patterns can be magnetized with an external permanent magnet or electromagnet (Deng et al. 2002; Do et al. 2004; Ino et al. 2008). Such design present similarities with iDEP structures mentioned above.

To obtain a high optical gradient field, the conventional optical tweezers use a standard Gaussian laser beam. The most commonly used sources in optical tweezing are red and near infrared (IR) lasers due to their excellent beam quality and technological maturity. These lasers are ideally suited to biological applications as a consequence of their low absorption in water and biological tissue. This highly beneficial property allows biological material to be manipulated whilst minimizing the potential for damage (Ashkin et al. 1987). The classical Gaussian beam can be used to trap a single particle at one time. In order to perform multiple manipulations of different cells, the traditional gaussian beams were replaced by NonGaussian laser beams. Among these Non-Gaussian modes, we can mention a Bessel beam which consists of a bright centre spot surrounded by series of concentric rings of decreasing intensity. Through the use of such a beam as optical tweezer, it is possible to trap and manipulate, within perpendicular two-dimensional planes, many different particles distributed over a distance of approximately $3 \mathrm{~mm}$ simultaneously (Paterson 2005). A Laguerre-Gaussian mode has also been used to obtain a rotating asymmetric intensity profile. It is characterized by an intensity maxima spiral around a dark centre core, that is, a 
zero on-axis intensity. The presence of this dark centre core of weak intensity allows the trapping of reflecting particles, absorbent particles or particles with a low dielectric constant that could be expelled or degraded by standard Gaussian laser beam (O'Neil et al. 2001). Other optical tweezers systems were created to enable the manipulation of several cells simultaneously. Among them, we can mention the Acousto-optic deflectors (AODs) that is a powerful tool enabling creation and control of several (up to 100) independent optical tweezers only by deflecting one incoming laser beam. The AODs appear to be excellent for two-dimensional optical landscapes (Vossen et al. 2004). However, this technique is not convenient for the trapping of very small particles having a too fast Brownian movement. An alternative consists in using networks of laser diodes (Applegate et al. 2004) or vertical cavity surface emitting laser (VCSEL) to trap and manipulate microparticles (Ogura et al. 2002) or biological cells (Flynn et al. 2002). However, they have a relatively low power input. The limitation is that only cells smaller than $10 \mu \mathrm{m}$ in size can be moved using the current VCSEL-driven optical tweezers. Other techniques use interference patterns with several laser beams to create several traps. MacDonald and al were able to build networks of threedimensional traps by using interference patterns created with two Laguerre-Gauss beams called "doughnut" (MacDonald and coworkers, 2002). These interference patterns generate four individual tweezers that can be at the same time translated in three dimensions in the space. To achieve true three-dimensional multiple optical traps, one must choose holographic optical tweezers (HOT). HOTs offer complete flexibility in manipulating multiple particles independently (Curtis et al. 2002). A last way used for trapping and optical manipulation of cells consists in using evanescent fields. The first experiment in this domain was realized by Kawata and Sugiura (Kawata et al. 1992). The manipulation of particles by evanescent fields opens interesting perspectives for the design of innovative and compact systems for the manipulation, the detection and the sorting of particles or biological objects. It is also a step towards systems of optical positioning with very strong precision. Indeed, the volumes of traps defined by propagated fields are intrinsically limited by the diffraction phenomenon in free space, which is not the case for evanescent waves.

As regards micro scale acoustic manipulation, cells are subjected to an ultrasonic standing wave (USW) field generated by one or more on-chip integrated transducers. The USW is commonly generated throughout the fluid comprised between two parallel vertical walls of a rectangular resonator chamber etched in silicon (Nilsson et al. 2004) or glass (Laurell et al. 2007; Evander et al. 2008). The ultrasonic transducer can be placed underneath the channel, in which case the USW field induced in the fluid is orthogonal to the direction of transmission. This will result in particles collecting in planes perpendicular to the actuator and parallel to the chamber walls. Another design option consists in placing the actuator at one of the resonator walls (Hill et al. 2002; Lilliehorn et al. 2005). In this case, the induced USW is parallel to the direction of transmission and cells collect in planes parallel to both the transducer and the chamber walls. Higher force magnitudes scan be obtained by scaling down the channel dimensions. Indeed, the axial PRF is proportional to the frequency, which increases as the channel size decreases. The typical operating frequency ranges from 100 $\mathrm{kHz}$ to $10 \mathrm{MHz}$, with corresponding channel dimensions comprised between a millimeter to a few tens of micrometers (Laurell et al. 2007). The pressure amplitude may also be increased to obtain more important forces by adjusting the actuation voltage. The maximum applicable voltage is limited by the heating of the device, which is a particular concern in cell handling applications (Evander et al. 2007). 


\section{Applications}

\subsection{Cell positioning}

Cell immobilization methods relying on contactless manipulation tools were recently reviewed by Johann (Johann 2006). As there is a wide range of applications requiring precise cell positioning, we have chosen to illustrate the possibilities offered by the different methods by focusing on the particularly challenging operation of cell arraying. Current diagnostic techniques or pharmacological screening require parallel analysis of millions of cells. Improving the technology for gathering cells into clumps make it possible to treat and control them differently while keeping them under observation. Precise positioning of individual cells is also required in many situations.

One of the advantages of DEP and laser tweezers is their high spatial accuracy suitable for 3D manipulation of individual cells. Recent technological advances have enabled the fabrication of micro scale magnetic structures with well-defined features, which improved the spatial accuracy of magnetic manipulation tools. Acoustic manipulation is well suited for simultaneous handling of large groups of cells and less adapted to localized 3-D positioning of single cells, due to diffraction and absorption limitations for focused highfrequency ultrasonic field (Wiklund et al. 2006).

Figure 5 shows that both dielectrophoretic and diamagnetic trapping can be used to perform arraying of single cells or small groups of unlabelled cells. In the first case (fig. 5a), positioning accuracy is ensured by the use of quadrupole electrode sets symmetrically driven. Under $\mathrm{nDEP}$, cells were repelled toward the center of the interelectrode gaps (Frénéa et al. 2003). In the second case (fig. 5b.), diamagnetic yeast cells were placed in a medium enriched in paramagnetic ions. They were confined at regularly spaced magnetic traps produced by permanent micro magnets under the effect of negative magnetophoresis, without any contact with the surface. Cell levitation height was estimated to $8 \mu \mathrm{m}$ by simulation (Frenea-Robin et al. 2008).
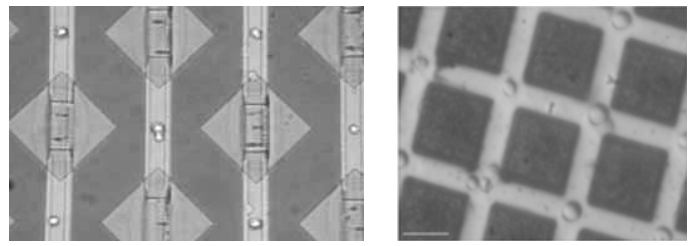

Fig. 5. a. Jurkat cells trapped at electric field minima by nDEP. b. 3-D trapping of yeast cells above an array of CoPt permanent micromagnets.

Compared with DEP, the use of permanent micro magnets offers the advantage to prevent Joule heating, but also poses the problem of later cell displacement, due to the static nature of the field. Diamagnetic levitation of micro droplets in air could also be performed with such micro magnet arrays, while nDEP required for dielectric levitation is non efficient in air. Tanase also described utilization of a 2-dimensional array of magnetized permalloy micro-ellipses for drawing magnetically labelled cells toward the tip of micro-ellipses and forming rows of cells (Tanase et al. 2005).

Lilliehorn et al. proposed to perform dynamic arraying of cells in a micro fluidic channel using an array of integrated ultrasonic micro transducers (Lilliehorn et al. 2005). This 
concept was validated on a one-dimensional array. The lateral extension of each trapping site was determined by the corresponding micro transducer dimensions $(0.8 \mathrm{~mm} \times 0.8 \mathrm{~mm})$. Haake and coworkers have proposed a method for the controlled positioning of cells in one or two dimensions (i.e. in lines or points) based on the use of ultrasonic forces in a millimeter-scale device (Haake et al. 2004; Haake et al. 2005). To apply these forces, a sound field was generated into a layer of fluid comprised between a vibrating glass plate situated on top of the device and a reflecting surface at the bottom. Activating a piezo-transducer attached at one plate edge resulted in the formation of a one-dimensional standing wave in the plate. Therefore, an acoustic wave propagated between the plate and the reflecting surface. This induced in turn a 2-D standing-wave sound field in the intermediate liquid layer, resulting in cells forming bands parallel to the activated transducer. When two orthogonal transducers were activated, a 3-D sound field was induced in the fluid layer. As a result, cells were collected on a grid of oval points orientated in zigzag patterns dictated by the contours of minimum force potential. In order to improve cell confinement, Neild used another approach to generate the required field. Two orthogonally aligned strip electrodes were defined in the metallic coating of a piezoelectric actuator, which was located beneath the fluid cavity etched in a silicon wafer. These electrodes could be addressed individually to create two orthogonal ultrasonic fields. A grid of well-confined circular cell clumps could be obtained when two signals with slightly different frequencies were applied to the electrodes (Neild et al. 2006).

Optical trapping can also be used to assemble and organize cells in order to form a group of cells into a new structure with predefined configurations. Assembling and organizing cells require the utilization of computer-controlled SLMs (Spatial Light Modulators) built on holographic optical tweezers. This technique allows the manipulation of cells both laterally and axially over several tens of micrometres so that cells can be rearranged into $2 \mathrm{D}$ and $3 \mathrm{D}$ configurations. Therefore, Flynn and co-workers demonstrated 2D trapping of $2 \times 2$ arrays of Yeast cells and human red blood cells using a single VCSEL beam optical tweezers. They also indicated that maximum force in the VCSEL optical trap varies depending on the size and relative index of refraction of the trapped cells, as well as the scales of optical power (Flynn et al. 2002). Jordan et al. succeeded to fixe E. coli cells in liquid gelatine at predefined positions in a 3D structure (Jordan 2005). They anticipate that the ability to form such viable three dimensional structures will open a wide range of future applications, including the arrangement of various cell types in complex architectures, as motifs for promoting tissue differentiation and growth within the field of cell engineering. Akselrod et al. demonstrated the assembly of 3D heterotypic microarrays consisting of a Swiss 3T3 mouse fibroblast surrounded by a ring of Pseudomonas aeruginosa bacteria by using arrays of timemultiplexed holographic optical tweezers (Akselrod et al. 2006).

\subsection{Cell sorting}

\section{a. Binary separation}

A binary separation of a mixed cell population can be achieved if the medium properties are balanced in such a way that the contrast factors of the two cell types are of opposite signs. Indeed, the two fractions can be spatially separated, one being driven in the direction of the field gradient, and the other at the opposite. As an example, viable and non-viable cells were selectively isolated using positive and negative dielectrophoretic forces which respectively trapped them at the electrode edges or repelled them form the electrodes 
(Markx et al. 1994). To perform such separation, the field frequency must fall between the DEP crossover frequencies of the two cell types. Physical separation of the two subpopulations is achieved by superimposing a liquid flow above the electrode array. Cells trapped at field minima (nDEP) can be eluted towards a device outlet, while those experiencing $\mathrm{pDEP}$, held by a stronger force, remain attracted to the electrodes until the field is switched-off (Wang et al. 1993). For increased efficiency, flow separation can also be operated as a continuous process. In this case, the suspension is continuously flowing above the electrodes, and the cells are either trapped on the array by pDEP or collected at the outlet. The processing speed is limited by the ability of the electrodes to remove a passing particle from the fluid stream and retain it against the action of viscous drag (Hughes 2002). More than two particle sub-populations can be differentiated by performing several steps of frequency tuning and repeating the separation process.
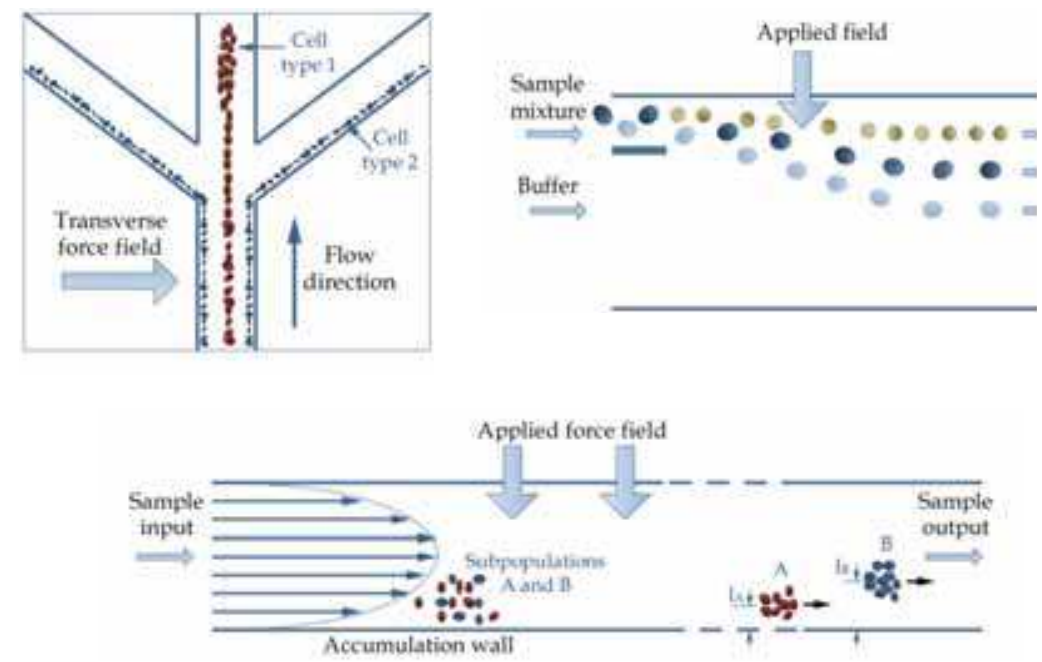

Fig. 6. a. and b. Continuous flow sorting based on the force sign (a) and/or magnitude (b), top view. c. Schematic representation of FFF (cross view). Sample B moves faster through the channel than $A$ since $l_{B}>l_{A}$. Separation is performed in the flow direction.

Doh presented a continuous cell separation chip using three planar electrodes in a separation channel, where the pDEP cells moved away from the central streamline while the $\mathrm{nDEP}$ cells remained in the central streamline. The laminar nature of the flow enabled the collection of cell fractions through different outlets (Doh et al. 2005).

Using a comparable design (fig. 6a), Laurell and coworkers demonstrated acoustic binary separation of red blood cells $(\phi>0)$ and lipid microemboli $(\phi<0)$ (Nilsson et al. 2004; Petersson et al. 2005; Laurell et al. 2007). The RBCs accumulated into the channel midplane corresponding to the USW pressure node, while the lipid particles where driven towards the anti-nodal planes at the sidewalls.

Binary separation based on optical forces can be achieved via either the intrinsic properties of the cells, such as size, shape and refractive index with and without fluid flow. For 
instance, the Bessel beam has been used for flow-free optical separation of lymphocytes from erythrocytes using the difference in their shape (Paterson 2005). Therefore, the biconcave-shaped erythrocytes locked into outer rings of the Bessel beam, while spherically shaped lymphocytes rapidly moved directly to the beam centre and they were collected from the centre core.

Despite their simplicity and high efficiency, binary separation techniques are not applicable in all cases. The forces experienced by the two cell types must be of different signs and have a significant magnitude. This is not achievable if the cell properties are not sufficiently different, as it becomes difficult to adjust the medium properties in between.

\section{b. Separation of polydisperse mixtures}

When more than two cell types must be separated, it is possible to differentiate them based on the force magnitude. Such strategy has been employed in cell deflectors and Field Flow Fractionation (FFF) devices. The force magnitude depends on the particle size and on the contrast factor amplitude.

\section{- Cell deflectors}

The inherent laminar nature of micro scale fluid dynamics has enabled the design of new continuous-flow cell separators. A classical continuous flow sorter design is represented on figure 6.b. The particles in the mixture respond differently to the applied force field and are thus deflected from the direction of flow, which enable to collect them at different outlets.

Petersson et al. validated the concept of free flow acoustophoresis (FFA), where particle separation is achieved by acoustic standing wave force-induced lateral displacement in the laminar flow of a micro channel. A $\lambda / 2$ USW generated between the side walls of the channel using a piezoceramic actuator gave rise to an acoustic force field perpendicular to the flow direction. At controlled flow rate, the particles that were most affected by the axial PRF reached the midplane of the channel while the others were dispersed between the sidewalls and the center. As the axial PRF is proportional to the particle volume, polystyrene particles sharing the same mechanical properties could be separated according to their size, which ranged from 2 to $10 \mu \mathrm{m}$ (Petersson et al. 2007).

Cell separation using nDEP deflexion has also been reported recently by Hu. Cells introduced in a micro fluidic channel were deflected by the electric field into the collection stream if they were tagged with a dielectrophoretically responsive label or directed to the waste outlet otherwise (Hu et al. 2005).

Magnetic separation of cells can be achieved by labelling a specific cell subpopulation with magnetic particles through antigen-antibody interactions. Therefore, magnetically tagged cells can be separated from non-magnetic ones in continuous flow by applying magnetic forces perpendicularly to the flow direction in order to deflect cells into different trajectories. These systems require the creation of strong magnetic fields inside the micro fluidic channel. Inglis et al. described separation of magnetically labelled WBCs from blood cells by using nickel stripes integrated on a micro channel as magnetic deflector (Inglis et al. 2004). Xia et al succeeded to extract magnetically labelled E. coli from RBCs thanks to a strong magnetic field gradient generated by micro comb structures of $\mathrm{NiFe}$ integrated in a micro channel (Xia et al. 2006). Berger used ferromagnetic wires oriented at an angle of $45^{\circ}$ to the flow of cells to develop a new magnetic sorting device. The sum of the hydrodynamic force and the magnetic field gradient created by wires constrains labelled cells to move along the magnetic wires where unlabelled cells flow into the central direction (Berger et al. 
2001). Pamme et al. described separation of magnetic particles from non-magnetic ones in a flat separation chamber composed by a number of inlet and outlet, just by applying a magnetic field gradient perpendicularly to the direction of the flow. The magnetic deflection of cells depends on their magnetic susceptibilities and their size (Pamme 2006).

Optical deflectors have been also used for cell sorting and are based on targeting the specific subpopulation of cells with fluorescent markers (Dholakia et al. 2007). The mechanism for cell sorting is very similar to that of macroscopic FACS machines (Givan 2001). In micro fluidic regime, cells are chosen via an external decision through the fluorescence signal from the detection region. Optical force is used as an optical switch for cell deflection. Buican et al. were the first to use a deflection beam for cell sorting by deflecting out cells of interest into a reservoir of choice (Buican et al. 1987). Later, $\mathrm{T}$ and Y-types of micro channel junctions were designed and coupled with optical tweezers for trapping and delivering several target cells to a collection reservoir simultaneously (Ozkan et al. 2003; Enger et al. 2004). Optical separation can also be carried out by attaching silica microspheres to a specific subpopulation of cells via antibody-antigen binding. The cells with microspheres attached reacted to the optical landscape more strongly than cells without any attached beads due to the higher refractive index mismatch and accompanying optical forces(Dholakia et al. 2007). Within fluid flow, cells are sorted thanks to a size- and shape-dependent gradient force in competition with a size- and shape-dependent viscous drag force. Flowing particles through an optical potential energy landscape, such as two-dimensional (2D) or 3D interference pattern or holographically produced array, will facilitate the separation of cells according to their physical characteristics. MacDonald and coworkers demonstrated the separation of erythrocytes from lymphocytes based on shape difference by using an angled optical lattice (MacDonald et al. 2004).

\section{- Chromatographic separation}

Dielectrophoresis has also been widely applied as a force field in field-flow fractionation (FFF), a technique falling into the category of chromatographic separation methods. In FFF techniques, the field is applied at right angle to flow (fig. 6c) and serves to drive different types of particles into different stream laminae of the carrier fluid. As a result, different particles will travel at different velocities according to their distance from the bottom surface. Those particles, which are introduced to the channel inlet at the same point and time, will emerge from the outlet at different times according to their reaction to the applied field. In DEP-FFF, an electrode array is patterned onto the bottom surface and repels cells by nDEP towards a stable position. The resulting force on the particle results from both the nDEP effects and the buoyancy force. The levitation heights reached by different cell types (and therefore their velocity) will depend on both their dielectric properties (via $\operatorname{Re}\left(\mathrm{f}_{\mathrm{CM}}\right)$ ) and densities. DEP-FFF has enabled the fractionation of various cell types and was even utilized to detect the effect of the differential expression of a single gene in genetically identical cells (Gascoyne et al. 2004).

twDEP can also be used to establish the chromatograph of a cell mixture, by introducing the sample at one end of the electrode array and then forcing cells to move along the tracks. The cell velocity is related to the dielectric properties of the particles, via $\operatorname{Im}\left(\mathrm{f}_{\mathrm{CM}}\right)$. Hence some cell subpopulations will move more rapidly than others and will therefore be collected before at the opposite end. This technique is particularly interesting when cells present only slight differences. In such cases, long electrode arrays can be used to enable a clear separation of cell groups (Green et al. 1997). 
Chromatographic separation requires precise sample injection prior to processing, which constitutes one of its main drawbacks compared to continuous flow devices (Pamme 2007). In tw-DEP devices, to ensure that particles start from one end of the electrode array simultaneously, sample precollection can be performed using cDEP electrodes that trap the particles by pDEP at the beginning of the tracks (Cui et al. 2001).

In the case of magnetic FFF, a magnetic field gradient is used as an external force passing at right angles to the channel, perpendicularly to the flow. The interaction of hydrodynamic and magnetic forces is used to separate the particles as they are carried along a separation channel according to their magnetic susceptibility and size. The controllability of both the field or gradient in field strength and the carrier flow rate contribute to the versatility of FFF. Other works described utilization of a ferromagnetic wire at the axis of channel placed perpendicular to a magnetic field that magnetized the wire allowing thus separation of particles (Carpinoa et al. 2005). Mitsuhashi et al. embedded ferromagnetic wires in both channel walls in the direction of fluid flow. The wires in each wall lay opposite spaces between wires in the other. An external field of 3T was applied. They were able to show slight retention of some transition metal salts, although band spreading was extremely high (Mitsuhashi et al. 2002). Recently, Carpino et al. reported utilization of a quadrupole magnet to obtain a field strength and field gradient relatively constant throughout an helical channel. They succeeded to retain many of the magnetic nanoparticles of interest and refer to the technique as Quadrupole magnetic field-flow fractionation (MgFFF) (Carpinoa et al. 2005).

Cell sorting can also be achieved using a balance between the viscous drag experienced by cells in fluid flow and the scattering force of a counter-propagating laser beam, termed 'optical chromatography' by Imasaka (Imasaka et al. 1995). When cells flow along the channel, the scattering force pushes them into the focal region of the laser beam. The net force between the scattering and drag forces results in different equilibrium positions for different cells. This method can be used to separate a range of cells including blood cells (Kaneta et al. 1997), bacterial spores and cells (Hart et al. 2007).

\section{Comparison criteria}

All the contactless forces described above depend on a force factor that changes according to the volume of manipulated cells. The capability of DEP to handle dielectric particles in a large range of size permits to obtain the larger force range in comparison with other contactless forces. Optical force exhibits the smaller force range due to tight focusing of the laser beam limiting the area over which the optical traps can be created and restricting the cell sizes that can be trapped. Numerous studies tend to show that acoustic manipulation can be considered to be non-lethal to cells (Hultström et al. 2007; Petersson et al. 2007). However, the majority of actuators employed for generating contactless forces (conductors, laser beams, electromagnets and piezoelectric transducers) may cause thermal damage, which restricts possibilities for long time cell exposition. Otherwise, dielectrophoresis may cause membrane breakdown, as a strong AC field is applied and a transmembrane potential induced (Lurquin 1997). The technique of iDEP presented in section 3.2 offers the possibility to avoid electrode fouling, which may also cause harm to cells.

No heat damages are induced by permanent micro magnets, which do not interfere with the ion concentration in aqueous solutions in contrary to dielectrophoresis. Moreover, highly 
selective separation of cells can be obtained using magnetic forces thanks to the large differences between magnetic susceptibilities of the magnetic labelled cells and nonmagnetic ones. However, magnetophoresis has been limited until recently to cells that either have an intrinsic magnetic response or are tagged with magnetic beads but tagging protocols may not exist for all the cells of interest. DEP, acoustic and optic manipulations are generally based on tag-free protocols that do not require attaching cells to magnetic beads or other particles which are more attractive to biologists. Regarding elaboration of contactless manipulation systems, the integration of microelectrodes on microsystems remains easier than that of micro magnets. Optical tweezers are incorporated easily into a conventional microscope arrangement, the same objective lens being used for both focusing the laser beam and viewing the trapped object. Optical systems are suitable for single cell manipulation with high 3D precision but are not convenient for parallel manipulation owing to limited area over which the optical traps can be created. In the case of acoustic manipulation, the periodicity of the standing-wave can be exploited to handle many particles simultaneously. In many applications based on this technique, a single actuator is required to handle many cells simultaneously and generate a USW field throughout the whole channel. This may constitute an advantage over DEP, which uses a force field with short-range effects (Neild et al. 2007). But as a counterpart, until now, DEP has proven to offer more precision and versatility. Frequency-dependency of cell dielectric properties lends to DEP a dimension of discrimination that also constitutes an advantage of this widely used technique.

\begin{tabular}{|c|c|c|c|c|}
\hline Method & DEP & Magnetic & Optic & Acoustic \\
\hline Factor & $f_{C M}$ & $\Delta \chi$ & $Q$ & $\phi(\beta, \rho)$ \\
\hline $\begin{array}{c}\text { Force range } \\
(\mathrm{pN})\end{array}$ & $20-100000$ & $10-10000$ & $0.1-100$ & $0.1-1000$ \\
\hline $\begin{array}{c}\text { Separation } \\
\text { criteria }\end{array}$ & $\begin{array}{c}\text { Complex } \\
\text { permittivity }\end{array}$ & $\begin{array}{c}\text { Magnetic } \\
\text { susceptibility }\end{array}$ & Refractive index & $\begin{array}{c}\text { Density and } \\
\text { compressibility }\end{array}$ \\
\hline Advantages & $\begin{array}{c}\text { Frequency- } \\
\text { dependency }\end{array}$ & $\begin{array}{c}\text { Highly selective } \\
\text { separation }\end{array}$ & $\begin{array}{c}\text { Single cell 3D } \\
\text { manipulation } \\
\text { with high } \\
\text { precision }\end{array}$ & Long-range effects \\
\hline Disadvantages & $\begin{array}{c}\text { Joule heating and } \\
\text { modification of } \\
\text { transmembrane } \\
\text { potential }\end{array}$ & $\begin{array}{c}\text { Magnetic } \\
\text { labelling }\end{array}$ & $\begin{array}{c}\text { Photodamage and } \\
\text { thermal damage } \\
\text { Not possible in } \\
\text { optically opaque } \\
\text { systems }\end{array}$ & $\begin{array}{c}\text { Temperature } \\
\text { increase }\end{array}$ \\
\hline
\end{tabular}

Table 3. Comparison of DEP, magnetic, optic and acoustic manipulation methods.

As each of the techniques presented above presents its own advantages and drawbacks, a potential for future research seem to be the development of new hybrid systems. For instance, Wiklund et al. combined DEP, acoustic and viscous drag forces to perform both high-precision handling of individual cells and high-throughput handling of groups of cells (Wiklund et al. 2006). As the use of ultrasound seem to be more appropriate than electric field for long-term exposition, DEP can be used for short-term accurate positioning, prior to long-term retention by acoustic forces. 
By coupling optical force and dielectrophoresis, Chiou et al. presented for the first time a novel optoelectronic tweezers (OET) capable of trapping and transporting multiple biological cells (Chiou et al. 2004). This technique is based on focusing optical beam on a photoconductive surface to create a highly non-uniform electric field allowing optical addressing of dielectrophoresis forces with a spatial resolution of $\sim 1 \mu \mathrm{m}$. The optical intensity required is three orders of magnitudes lower than that of conventional optical tweezers. The OET does not require tight optical focusing and the trapping area can be tailored to match the cell size. It also offers the advantage of producing larger forces (several hundred picoNewtons) for cell manipulation (Choi et al. 2002; Hwang et al. 2008).

\section{Conclusion}

Various strategies of cell manipulation based on contactless forces were presented. Many contactless cell manipulations with batch and fluidic systems were described. Current research is concerned with the development of high performance micro fluidic systems in order to improve efficiency and throughput of cell manipulation with the aim to develop new solutions for clinical applications. Thanks to their similarities and differences, the contactless forces described in this chapter can be considered as elements of a toolbox that can be used according to the targeted cell species. The advantages and drawbacks of each contactless force can be evaluated as function of properties of manipulated cells as wells as working conditions. The combination of two or more manipulation tools will constitute a challenge for future research.

\section{References}

Akselrod, G. M., W. Timp, et al. (2006). "Laser-guided assembly of heterotypic threedimensional living cell microarrays " Biophys J 91: 3465-3473.

Applegate, R., J. Squier, et al. (2004). "Optical trapping, manipulation, and sorting of cells ans colloids in microfluidic systems with diode laser bars." Optics express 12(19): 4390-4398.

Ashkin, A., J. M. Dziedzic, et al. (1987). "Optical trapping and manipulation of single cells using infrared beams." Nature (London) 330: 769-771.

Balaev, A. E., K. N. Dvoretski, et al. (2001). "Refractive index of escherichia coli cells " Saratov Fall Meeting: Optical Technologies in Biophysics and Medicine III 4707: 253-260.

Berger, M., J. Castelino, et al. (2001). "Design of a microfabricated magnetic cell separator." Electrophoresis 22: 3883-3892.

Buican, T. N., M. J. Smyth, et al. (1987). "Automated single-cell manipulation and sorting by light trapping." Appl. Opt. 26: 5311-5316.

Burt, J. P. H., R. Pethig, et al. (1990). "Dielectrophoretic characterisation of Friend murine erythroleukaemic cells as a measure of induced differentiation." Biochimica et Biophysica Acta, 1034: 93-101.

Carpinoa, F., L. R. Moorea, et al. (2005). "Analysis of magnetic nanoparticles using quadrupole magnetic field-flow fractionation." Journal of Magnetism and Magnetic Materials 293: 546-552. 
Chiou, P. Y., W. Wong, et al. (2004). Cell adressing and trapping using novel optoelectronic tweezers. 17th IEEE International Conference on MEMS.

Choi, J.-W., J. Do, et al. (2002). Hybrid type on-chip magnetic particle separators for accurate positioning magnetic beads. Micro Total Analysis Systems, Nara, Japan.

Coakley, W. T. (1997). "Ultrasonic separations in analytical biotechnology." Trends in Biotechnology 15: 506-511.

Cui, L., D. Holmes, et al. (2001). "The dielectrophoretic levitation and separation of latex beads in microchips." Electrophoresis 22: 3893-3901.

Curtis, J. E., B. A. Koss, et al. (2002). "Dynamic holographic optical tweezers." Opt. Commun. 207: 169-175.

De Gasperis, G., X.-B. Wang, et al. (1998). "Automated electrorotation: dielectric characterization of living cells by real-time motion estimation." Meas.Sci.Technol. 9: 518-529.

Deng, T., M. Prentiss, et al. (2002). "Fabrication of magnetic microfiltration systems using soft lithography." Applied physics letters 80(3): 461-463.

Dholakia, K., W. M. Lee, et al. (2007). "Optical separation of cells on potential energy landscapes: enhancement with dielectric tagging." IEEE Journal of Selected Topics in Quantum Electronics 13(6): 1646-1654.

Do, J., J. Choi, et al. (2004). "Low-cost magnetic interdigitated array on a plastic wafer." Lab on a Chip 40: 3009-3011.

Doh, I. and Y.-H. Cho (2005). "A continuous cell separation chip using hydrodynamic dielectrophoresis (DEP) process." Sensors and Actuators A 121: 59-65.

Enger, J., M. Goksor, et al. (2004). "Optical tweezers applied to a microfluidic system." Lab Chip 4: 196-200.

Evander, M., L. Johansson, et al. (2007). "Noninvasive acoustic cell trapping in a microfluidic perfusion system for online bioassays." Anal Chem 79: 2984-2991.

Evander, M., A. Lenshof, et al. (2008). "Acoustophoresis in wet-etched glass chips." Anal Chem 80: 5178-5185.

Flynn, R. A., A. L. Birkbeck, et al. (2002). "Parallel transport of biological cells using individually addressable VCSEL arrays as optical tweezers. ." Sensors and Actuators B 87: 239-243.

Frénéa, M., S. P. Faure, et al. (2003). "Positioning living cells on a high-density electrode array by negative dielectrophoresis." Materials Science and Engineering: C 23: 597-603.

Frenea-Robin, M., H. Chetouani, et al. (2008). Contactless diamagnetic trapping of living cells onto a micromagnet array. IEEE Eng Med Biol Soc., Vancouver.

Gascoyne, P., C. Mahidol, et al. (2002). "Microsample preparation by dielectrophoresis: isolation of malaria." Lab Chip 2: 70-75.

Gascoyne, P., R. Pethig, et al. (1997). "Dielectrophoretic detection of changes in erythrocyte membranes following malarial infection." Biochem Biophys Acta 323: 240-252.

Gascoyne, P., J. Satayavivad, et al. (2004). "Microfluidic approaches to malaria detection." Acta Tropica 89: 357-369.

Gascoyne, P. R. C., F. F. Becker, et al. (1995). "Numerical analysis of the influence of experimental conditions on the accuracy of dielectric parameters derived from electrorotation measurements." Bioelectrochemistry and bioenergetics 36: 115-125.

Gascoyne, P. R. C., J. Noshari, et al. (1994). "Use of dielectrophoretic collection spectra for characterizing differences between normal and cancerous cells." IEEE transactions on industry applications 30: 829-834. 
Gascoyne, P. R. C. and J. V. Vykoukal (2004). "Dielectroporesis-based sample handling in general-purpose programmable diagnostic instruments." Proceedings of the IEEE 92(1): 22-40.

Givan, A. L. (2001). Flow cytometry: first principles, Wiley-Liss, USA.

Godin, M., A. K. Bryan, et al. (2007). "Measuring the mass, density and size of particles and cells using a suspended microchannel resonator." Applied physics letters 91.

Green, N. G., M. P. Hughes, et al. (1997). "Large area multilayered electrode arrays for dielectrophoretic fractionation." Microelectronic Engineering 35: 421-424.

Gupta, S. and D. L. Feke (1995). "Fractionation of mixed particulate solids according to compressibility using ultrasonic standing wave fields." Chemical Engineering Science 50(20): 3275-3284.

Haake, A. and J. Dual (2004). "Positioning of small particles by an ultrasound field excited by surface waves." Ultrasonics 42: 75-80.

Haake, A., A. Neild, et al. (2005). "Positioning, displacement and localization of cells using ultrasonic forces." Biotechnology and Bioengineering 92(1): 8-14.

Häfeli, U. O., R. Ciocan, et al. (2002). "Characterization of magnetic particles and microspheres and their magnetophoretic mobility using a digital microscopy method." European Cells and Materials 3: 24-27.

Han, K. H. and B. Frazier (2004). "Continuous magnetophoretic separation of blood cells in microdevice format." Journal of Applied Physics 96(10): 5797-5802.

Hart, S. J., A. Terray, et al. (2007). "Sample concentration using optical chromatography." Opt. Express 15: 2724-2731.

Hill, M., Y. Shen, et al. (2002). "Modelling of layered resonators for ultrasonic separation." Ultrasonics 40: 385-392.

$\mathrm{Hu}$, X., P. H. Bessette, et al. (2005). "Marker-specific sorting of rare cells using dielectrophoresis." PNAS 102(44): 15757-15761.

Huang, Y. and R. Pethig (1991). "Electrode design for negative dielectrophoresis." Meas.Sci.Technol.2: 1142-1146.

Huang, Y., X.-B. Wang, et al. (1999). "Membrane dielectric responses of human Tlymphocytes following mitogenic stimulation." Biochem Biophys Acta 1417: 51-62.

Huang, Y., X.-B. Wang, et al. (1995). "Electrorotational studies of the cytoplasmic dielectric properties of Friend murine erthroleukaemia cells." Phys. Med. Biol. 40: 1789-1806.

Hughes, M. P. (2002). "Strategies for dielectrophoretic separation in laboratory-on-a-chip systems." Electrophoresis 23: 2569-2582.

Hultström, J., O. Manneberg, et al. (2007). "Proliferation and viability of adherent cells manipulated by standing-wave ultrsaound in a microfluidic chip." Ultrasound in Medicine \& Biology 33(1): 145-151.

Hwang, H., Y.-J. Choi, et al. (2008). "Interactive manipulation of blood cells using a lensintegrated liquid crystal display based optoelectronic tweezers system." Electrophoresis 29(6): 1203-1212.

Imasaka, T., Y. Kawabata, et al. (1995). "Optical chromatography." Anal Chem 67: 1763-1765.

Inglis, D. W., R. Riehn, et al. (2004). "Continuous microfluidic immunomagnetic cell separation." Appl. Phys. Lett. 85: 5093-5095.

Inglis, D. W., R. Riehn, et al. (2006). "Microfluidic high gradient magnetic cell separation." Journal of Applied Physics 99(8): 08K101.1-08K101.3.

Ino, K., M. Okochi, et al. (2008). "Cell culture arrays using magnetic force-based cell patterning for dynamic single cell analysis." Lab on a Chip 8: 134-142. 
Inoue, T., R. Pethig, et al. (1988). "Dielectrophoretic behaviour of micrococcuslysodeikticus and its protoplast." Journal of electrostatics 21: 215-223.

Irimajiri, A., T. Hanai, et al. (1979). "A dielectric theory of multi-stratified shell model with its application to a lymphoma cell." J Theor Biol 78(2): 251-269.

Jaeger, M. S., K. Uhlig, et al. (2008). "Contact-free single-cell cultivation by negative dielectrophoresis." J.Phys.D, Apply.Phy 41.

Jin, Y. L., J. Y. Chen, et al. (2006). "Refractive index measurement for biomaterial samples by total internal reflection." Phys. Med. Biol. 51: 371-379.

Johann, R. M. (2006). "Cell trapping in microfluidic chips." Anal. Bioanal. Chem 385: 408-412.

Jones, T. B. (2003). "Basic theory of dielectrophoresis and electrorotation." IEEE Eng. Med. Biol. Magazine 33-42.

Jordan, P. (2005). "Creating permanent 3D arrangements if isolated cells using holographic optical tweezers " Lab Chip 5: 1224-1228.

Kaneta, T., Y. Ishidzu, et al. (1997). "Theory of optical chromatography." Anal Chem 69: 2701-2710.

Kawata, S. and T. Sugiura (1992). "Movement of micrometer-sized particles in the evanescent field of a laser beal." Optics Letters 17(11): 772-774.

Kimura, T., Y. Sato, et al. (2005). "Micropatterning of Cells Using Modulated Magnetic Fields." Langmuir 21: 830-830.

Kimura, T., M. Yamato, et al. (2004). "Particle Trapping and Undulation of a Liquid Surface Using a Microscopically Modulated Magnetic Field." 20: 572-574.

Lapizco-Encinas, B., B. A. Simmons, et al. (2004). "Insulator-based dielectrophoresis for the selective concentration and separation of live bacteria in water." Electrophoresis 25: 1695-1704.

Laurell, T., F. Petersson, et al. (2007). "Chip integrated strategies for acoustic separation and manipulation of cells and particles." Chem. Soc. Rev. 36: 492-506.

Lee, H., A. M. Purdon, et al. (2004). "Micromanipulation of biological systems with microelectromagnets." IEEE Transactions on magnetics 40(4): 2991-2993.

Lilliehorn, T., M. Nilsson, et al. (2005). "Dynamic arraying of microbeads for bioassays in microfluidic channels." Sensors and Actuators B 106: 851-858.

Lurquin, P. F. (1997). "Gene transfer by electroporation." Molecular Biotechnology 7: 5-35.

MacDonald, M. P., S. Neale, et al. (2004). "Cell cytometry with a light touch: sorting microsocpic matter with an optical lattice." Journal of Biological Regulators and Homeostatic Agents: 200-205.

MacDonald, M. P., L. Paterson, et al. (2002). "Creation and manipulation of threedimensional optically trapped structures." Science 296(5570): 1101-1103.

Markx, G. H., M. S. Talary, et al. (1994). "Separation of viable and non-viable yeast using dielectrophoresis." J. Biotechnol. 32(1): 29-37.

Masuda, S., M. Washizu, et al. (1989). "Novel method of cell fusion in field constriction area in fluid integrated circuit." IEEE Trans. Industry Applicat. 25(4): 732-737.

Mitsuhashi, K., R. Yoshizaki, et al. (2002). "Retention of ions in a magnetic chromatograph using high-intensity and high gradient magnetic fields." Sep. Sci. Technol. 37: 36353645.

Moore, L. R., S. Milliron, et al. (2004). "Control of magnetophoretic mobility by susceptibility-modified solutions as evaluated by cell tracking velocimetry and continuous magnetic sorting." Anal. Chem. 76: 3899-3907. 
Moore, L. R., M. Zborowski, et al. (2000). "The use of magnetite-doped polymeric microspheres in calibrating cell tracking velocimetry." J. Biochem. Biophys. Methods 44: 115-130.

Müller, T., G. Gradl, et al. (1999). "A 3-D microelectrode system for handling and caging single cells and particles." Biosensors and bioelectronics 14: 247-256.

Neild, A., S. Oberti, et al. (2007). "Design, modeling and characterization of microfluidic devices for ultrasonic manipulation." Sensors and Actuators B 121: 452-461.

Neild, A., S. Oberti, et al. (2006). "Simultaneous positioning of cells into two-dimensional arrays using ultrasound." Biotechnology and Bioengineering 97(5): 1335-1339.

Nieminen, T., V. Loke, et al. (2007). "Optical tweezers computational toolbox." J.Opt.A: Pure Appl. Opt. 9: S196-S203.

Nilsson, A., F. Petersson, et al. (2004). "Acoustic control of suspended particles in micro fluidic chips." Lab Chip 4: 131-135.

O'Neil, A. T. and M. J. Padgett (2001). "Axial and lateral trapping efficiency of laguerregaussian modes in inverted optical twezeers." Optics Communications 193: 45-50.

Ogura, Y., S. Nobuhiro, et al. (2002). "Optical levitation and translation of a microscopic particle by use of multiple beams generated by vertical-cavity-surface-emitting laser arrays sources." Applied Optics 41: 5645-5653.

Ozkan, M., M. Wang, et al. (2003). "Optical manipulation of objects and biological cells in microfluidic devices." Biomed. Microdevices 5: 61-67.

Pamme, N. (2006). "Magnetism and microfluidics." Lab on a Chip 6: 24-38.

Pamme, N. (2007). "Continuous flow separations in microfluidic devices." Lab on a Chip 7: 1644-1659.

Park, K., J. Jang, et al. (2008). "'Living cantilever arrays' for characterization of mass of single live cells in fluids." Lab Chip 8: 1034-1041.

Park, S., M. Koklu, et al. (2009). "Particle trapping in high-conductivity media with electrothermally enhanced negative dielectrophoresis." Anal Chem 81(6): 2303-2310.

Paterson, L. (2005). "Light-induced cell separation in a tailored optical landscape " Appl. Phys. Lett. 87.

Petersson, F., L. Aberg, et al. (2007). "Free flow acoustophoresis : microfluidic-based mode of particle and cell separation." Anal. Chem. 79: 5117-5123.

Petersson, F., A. Nilsson, et al. (2005). "Continuous separation of lipid particles from erythrocytes by means of laminar flow and acoustic standing wave forces." Lab on a Chip 5: 20-22.

Pethig, R., Y. Huang, et al. (1992). "Positive and negative dieletrophoretic collection of colloidal particles using interdigitated castellated microelectrodes." J.Phys.D, Apply.Phy 24: 881-888.

Pohl, H. A. (1958). "Some effects of non uniform fields on dielectrics." J. Apply. Phys. 29: 1182-1188.

Price, J. A. R., J. P. H. Burt, et al. (1988). "Applications of a new optical technique for measuring the dielectrophoretic behaviour of micro-organisms." Biochimica et Biophysica Acta, 964: 221-230.

Ramadan, Q., D. P. Poenar, et al. (2009). "Customized trapping of magnetic particles." Microfluid Nanofluid 6: 53-62.

Rappaz, B., P. Marquet, et al. (2005). "Measurement of the integral refractive index and dynamic cell morphometry of living cells with digital holographic microscopy." Optics Express 13: 9361-9373. 
Ratanachoo, K., P. R. C. Gascoyne, et al. (2002). "Detection of cellular responses to toxicants by dielectrophoresis." Biochem Biophys Acta 1564: 449-458.

Safarik, I. and M. Safarikova (1999). "Use of magnetic techniques for isolation of cells." Journal of Chromatography B 722: 33-53.

Sato, S., M. Ishigure, et al. (1991). "Optical trapping and rotational manipulation of microscopic particles and biological cells using higher-order mode Nd:YAG laser beams." Electron. Lett 27: 1831-1832.

Saxena, V. (2003). "Polystyrene Microparticles as Handles for Optical Manipulation of CATH.a Cells" SURJ 2: 39-42.

Schenck, J. F. (2000). "Safety of strong, static magnetic fields." Journal of magnetic resonance imaging 12: 2-19.

Seger, U., S. Gawad, et al. (2004). "Cell immersion and cell dipping in microfluidic devices." Lab Chip 4: 148-151.

Sudsiri, J., D. Wachner, et al. (2007). "On the temperature dependance of the dielectric membrane properties of human red blood cells." Bioelectrochemistry 70: 134-140.

Svoboda, K. and S. M. Block (1994). "Biological applications of optical forces." Annu.Rev.Biophys.Biomol.Struct. 23: 247-285.

Tanase, M., E. J. Felton, et al. (2005). "Assembly of multicellular constructs and microarrays of cells using magnetic nanowires." Lab on a Chip 5: 598-605.

Toubal, M., M. Asmani, et al. (1999). "Acoustic measurement of compressibility and thermal expansion coefficient of erythrocytes." Phys. Med. Biol. 44: 1277-1287.

Tsutsui, H. and C.-M. Ho (2009). "Cell separation by non-inertial force fields in microfluidic systems." Mechanics Research Communications 36: 92-103.

Vossen, D. L. J., A. Van der Horst, et al. (2004). "Optical tweezers and confocal microscopy for simultaneous three-dimensional manipulation and imaging in concentrated colloidal dispersions." Rev. Sci. Instrum. 75: 2960-2970.

Wang, X.-B., M. P. Hughes, et al. (1995). "Non-uniform spatial distributions of both magnitude and phase of AC electric fields determine dielectrophoretic forces." Biochem Biophys Acta 1243: 185-194.

Wang, X.-B., H. Y, et al. (1994). "A unified theory of dielectrophoresis and travelling wave dielectrophoresis." J.Phys.D, Apply.Phy 27: 1571-1574.

Wang, X. B., Y. Huang, et al. (1993). "Selective dielectrophoretic confinement of bioparticles in potential energy wells." J.Phys.D, Apply.Phy 26: 1278-1285.

Wanichapichart, P., S. Bunthawin, et al. (2002). "Determination of cell dielectric properties using dielectrophoretic technique." ScienceAsia 28: 113-119.

Wiklund, M., C. Günther, et al. (2006). "Ultrasonic standing wave manipulation technology integrated into a dielectrophoretic chip." Lab on a Chip 6: 1537-1544.

Winkleman, A., K. L. Gudiksen, et al. (2004). "A magnetic trap for living cells suspended in a paramagnetic buffer." Applied physics letters 85(12): 2411-2413.

Xia, N., T. P. Hunt, et al. (2006). "Combined microfluidic-micromagnetic separation of living cells in continuous flow." Biomedical Microdevices 8: 299-308.

Yang, J., Y. Huang, et al. (1999). "Dielectric properties of human leukocyte subpopulations determined by electrorotation as a cell separation criterion." Biophysical Journal 76(6): 3307-3314. 


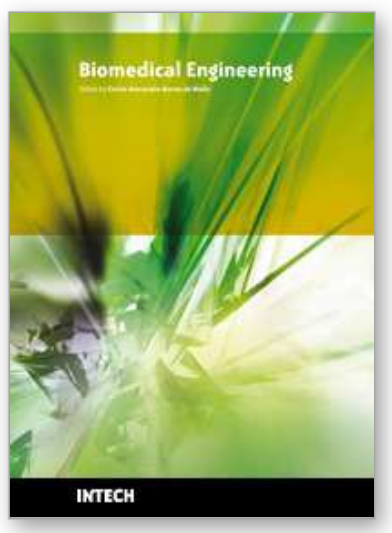

\author{
Biomedical Engineering \\ Edited by Carlos Alexandre Barros de Mello
}

ISBN 978-953-307-013-1

Hard cover, 658 pages

Publisher InTech

Published online 01, October, 2009

Published in print edition October, 2009

Biomedical Engineering can be seen as a mix of Medicine, Engineering and Science. In fact, this is a natural connection, as the most complicated engineering masterpiece is the human body. And it is exactly to help our "body machine" that Biomedical Engineering has its niche. This book brings the state-of-the-art of some of the most important current research related to Biomedical Engineering. I am very honored to be editing such a valuable book, which has contributions of a selected group of researchers describing the best of their work. Through its 36 chapters, the reader will have access to works related to ECG, image processing, sensors, artificial intelligence, and several other exciting fields.

\title{
How to reference
}

In order to correctly reference this scholarly work, feel free to copy and paste the following:

Frénéa Marie and Haddour Naoufel (2009). On-chip Cell Positioning and Sorting Using Contactless Methods: a Comparison between Different Force-fields, Biomedical Engineering, Carlos Alexandre Barros de Mello (Ed.), ISBN: 978-953-307-013-1, InTech, Available from: http://www.intechopen.com/books/biomedicalengineering/on-chip-cell-positioning-and-sorting-using-contactless-methods-a-comparison-between-differentforce-

\section{INTECH}

open science | open minds

\section{InTech Europe}

University Campus STeP Ri

Slavka Krautzeka 83/A

51000 Rijeka, Croatia

Phone: +385 (51) 770447

Fax: +385 (51) 686166

www.intechopen.com

\section{InTech China}

Unit 405, Office Block, Hotel Equatorial Shanghai

No.65, Yan An Road (West), Shanghai, 200040, China 中国上海市延安西路65号上海国际贵都大饭店办公楼 405 单元 Phone: +86-21-62489820

Fax: $+86-21-62489821$ 
(C) 2009 The Author(s). Licensee IntechOpen. This chapter is distributed under the terms of the Creative Commons Attribution-NonCommercial-ShareAlike-3.0 License, which permits use, distribution and reproduction for non-commercial purposes, provided the original is properly cited and derivative works building on this content are distributed under the same license. 